\title{
Moxibustion versus diclofenac sodium gel for the treatment of knee osteoarthritis: a double-blinded, double-placebo, randomized controlled trial
}

\section{Ling Luo}

Chengdu University of Traditional Chinese Medicine

Mo Liao

Chengdu University of Traditional Chinese Medicine https://orcid.org/0000-0003-3257-1775

Jian-Ying Zhou

Yancheng Traditional Chinese Medicine Hospital Affiliated to Nanjing University

Jia-Xi Peng

Chengdu University of Traditional Chinese Medicine

\section{Xiang Wang}

The First Affiliated Hospital of Xi'an Medical University

\section{Cai-Rong Zhang}

Nanjing Hospital of Traditional Chinese Medicine

\section{Ling-Ling Guo}

Zibo Central Hospital

Min Liu

Pi County People's Hospital

Jia-Ping Wang

Pi County Hospital of Traditional Chinese Medicine

Xiao-Xia Han

Xinjin Hospital of Traditional Chinese Medicine

Sheng-Feng Lu

Nanjing University of Traditional Chinese Medicine

Tian-Shu Hou

Chengdu First People's Hospital

Ping Du

Medical Center Hospital of Qionglai City

Jing-Jing Zhao

Sichuan Second Hospital of Traditional Chinese Medicine

Jia Zuo

Affiliated Hospital of Shaanxi University of Chinese Medicine

Jing-Zhu Chen 
Chengdu University of Traditional Chinese Medicine

\section{Bi-Qing Huang}

Chengdu University of Traditional Chinese Medicine

Jia Peng

Chengdu University of Traditional Chinese Medicine

\section{Guo-Ping Ran}

Chengdu University of Traditional Chinese Medicine

\section{Lin-Lin Zhu}

Chengdu University of Traditional Chinese Medicine

\section{Hai-Yan Yin}

Chengdu University of Traditional Chinese Medicine

\section{Qiao-Feng Wu}

Chengdu University of Traditional Chinese Medicine

\section{Cheng-Shun Zhang}

Chengdu University of Traditional Chinese Medicine

\section{Peng Lv}

Chengdu University of Traditional Chinese Medicine

\section{Yong Tang ( $\square$ tangyong@cdutcm.edu.cn )}

https://orcid.org/0000-0002-2543-066X

\section{Shu-Guang Yu}

Chengdu University of Traditional Chinese Medicine

\section{Research}

Keywords: Moxibustion, Knee osteoarthritis, Double-blinded, Double-placebo, Randomized controlled trial

Posted Date: May 13th, 2020

DOI: https://doi.org/10.21203/rs.3.rs-20609/v1

License: (c) (1) This work is licensed under a Creative Commons Attribution 4.0 International License. Read Full License 


\section{Abstract}

\section{Objective}

The purpose of this study was to evaluate the difference of efficacy between moxibustion (experimental group) and local diclofenac sodium gel (control group) in the treatment of knee osteoarthritis (KOA).

\section{Methods}

A multicenter, double-blinded, double-placebo, randomized controlled clinical trial was conducted in this research. 144 participants with knee osteoarthritis were randomly divided into two groups according to 1:1 ratio. The participants in the experimental group were treated with active Moxibustion plus placebo gel, whereas the control group was given diclofenac sodium gel plus placebo moxibustion. Moxibustion treatment was administrated at 3 acupoints (EX-LE4, ST35, ST36) for 4 weeks in 12 sessions. The entire trial cycle lasted 13 weeks, including 1 week at baseline, 4 weeks at treatment and 8 weeks at follow-up. Diclofenac sodium gel or placebo gel at a dose of $4 \mathrm{~g}$ per knee were applied three times per day for 4 weeks. The primary outcome measures were the changes in the Western Ontario and McMaster Universities Osteoarthritis Index (WOMAC). Secondary outcome measures included changes in other subscales of WOMAC (pain, stiffness and function), visual analogue scale (VAS) and participant overall assessment (PGA).

\section{Results}

The analysis showed that both the experimental group and the control group at 2 weeks, 4 weeks, 8weeks and 12 weeks were statistically significant compared with baseline $(P<0.05)$. There was no significant difference between the two groups in the first four weeks $(P>0.05)$. At 8 weeks, WOMAC total score $(95 \%$ $\mathrm{Cl}, 4.3$ [0.5 to 8.1], $\mathrm{P}=0.03)$, pain score $(95 \% \mathrm{Cl}, 1.0$ [0.2 to 1.9], $\mathrm{P}=0.02)$ and functional score $(95 \% \mathrm{Cl}, 3.0$ [0.1 to 5.8], $P=0.04)$ the control group are better than the experimental group $(P<0.05)$. At 12 weeks, there were no significant difference between the two groups $(P>0.05)$.

\section{Conclusions}

During the treatment, moxibustion has the same therapeutic effect as local diclofenac sodium gel, which can alleviate the pain of KOA participants to different degrees, rigidity and dysfunction. The therapeutic effect of it can basically maintained until 2 months after treatment. Local diclofenac sodium gel was superior to moxibustion in relieving knee pain and knee dysfunction at 8 weeks.

Trial registration number Clinical Trials.gov, NCT02769572.

Date of trial registration May 2016

$\bigotimes$ These authors contributed equally to this work. 


\section{Introduction}

Knee osteoarthritis (KOA) is a common chronic disease, with the main manifestations of pain, swelling and dysfunction of the joint. KOA affects the functional activities and quality of life of the elderly seriously [1], and is one of the main diseases of chronic disability[2].

For the treatment of KOA, non-steroidal anti-inflammatory analgesic drugs which have become an important part of the treatment of KOA are the main treatment of western medicine. Oral non-steroidal anti-inflammatory drugs (NSAIDs) have become an important part of the treatment of KOA. However, these drugs have a significant dose-related risk of gastrointestinal, cardiovascular and renal adverse events[3-6]. As a first-line alternative to drugs and a relatively new option for relieving knee pain, topical NSAIDs have the same efficacy as oral NSAIDs[7]. Currently, diclofenac as one of the topical NSAIDs, is considered by the European and American markets as an effective and safe topical medicine, such drugs can be used as the preferred drug for KOA pain[8], [9], [10], [11].

Moxibustion is one of the characteristics of Traditional Chinese Medicine treatments, which is a kind of natural therapies to treat the disease and keep health by burning herbal (Mugwort or Moxa) and stimulating acupoints[12]. In the clinic, moxibustion is widely used to treat various diseases and their indications, including OA[13]. The evidence shows that pain can be alleviated and the life quality can be improved after intervention by moxibustion therapy[14, 15]. Modern studies have found that moxibustion can reduce the role of local inflammation and repair of articular cartilage cells by inhibiting the expression of inflammatory reaction cytokines, reducing the content of enzymes associated with articular cartilage metabolism and activating endogenous opioid substances in the body [16], [17], [18]. Clinical studies have shown that moxibustion is effective in the treatment of KOA[19], [20],[ 21]. In a recent systematic review including 13 randomized controlled trials (RCTs) with a total of 1309 participants, the effects of moxibustion on the target population were found to be almost equal to the effects of oral drug and intra-articular injection[22]. The most recent systematic review and meta-analysis of moxibustion in the treatment of KOA show that moxibustion, compared with sham moxibustion and oral drugs, is effective for pain reduction and symptom management in KOA[23]. However, due to the inappropriate placement of control groups and the lack of appropriate double-blind methods, the risk of bias is high in most of the studies included because of the low quality of methodologies. Therefore, the role of moxibustion in $\mathrm{KOA}$ is still a controversial issue.

For these reasons, it is necessary to design a rigorous trial to evaluate the difference of efficacy between moxibustion and local diclofenac sodium gel in the treatment of KOA. Because of the particularity of moxibustion therapy, it is relatively difficult to make blind methods clinically, so we have implemented a rigorous double-blind, double-placebo, randomized controlled trial to observe the exact effect between moxibustion and local diclofenac sodium gel in treating KOA.

\section{Methods}




\section{Setting and Study Population}

We performed a multi-center, randomized, double blinded clinical trial for 13 weeks from May 2016 to July 2018. The trial protocol adhered to the CONSORT (Consolidated Standards of Reporting Trails) and STRICTA (Standards for Reporting Interventions in Clinical Trials of Acupuncture) guidelines[24]. The protocol was published previously[25]. This research has been approved by the Sichuan Regional Ethics Review Committee (permission number 2015KL-014).

All participants gave their oral and written informed consent. Participants mainly recruited to outpatient or inpatients in multiple clinical centers participated in this trial: Affiliated Hospital of Chengdu University of Traditional Chinese Medicine (TCM); Chengdu First People's Hospital; Sichuan Second Hospital of TCM; Sport Hospital Attached to Chengdu Sport Institute; Sichuan Orthopaedic Hospital; Pi Country People's Hospital; Xinqiao Hospital, Third Military Medical University; Xinjin Hospital of TCM; Medical Center Hospital of Qionglai City; Chongqing Traditional Chinese Medicine Hospital; Affiliated Hospital of Shaanxi University of Chinese Medicine; Xi'an Honghui Hospital; Nanjing Hospital of TCM; Affiliated Hospital of Nanjing University of TCM and Central Hospital of ZiBo. Fifteen moxibustion operators took part in the study.

The inclusion criteria were as follows: (1) male or female gender, aged between 40 and 75 years and having KOA diagnosed according to American College of Rheumatology criteria5[26]; (2) radiological confirmation of OA in one or both knees (Kellgren-Lawrence score of 2 or 3); (3) had knee pain for a duration of more than 3 months; (4) the average severity of knee pain being at least 3 points on a 10point visual analogue scale (VAS); (5) willingness to be randomly assigned and comply with our study protocol; and (6) agreed to sign the consent form. Participants excluded from the research if they: (1) have pain in the knee that may be caused by inflammatory, malignant, autoimmune disease or traumatic injury; (2) have serious diseases including cancer, uncontrolled hypertension, diabetes mellitus requiring insulin injection; life-threatening cardiovascular or neurological events; chronic respiratory disease; bleeding disorders; clinically active renal, hepatic or peptic ulcer diseases and serious mental diseases; (3) have undergone knee replacement surgery or arthroscopy of the affected knee within the past year, or received steroid or hyaluronic acid injection in the knee joints within the previous 3 months; (4) have received physiotherapy including acupuncture or cupping for knee pain during the previous 4 weeks; (5) have had previous experience with moxibustion treatment; (6) are pregnant or lactating women; and (7) are participating in another clinical trial.

\section{Interventions}

Moxibustion operators are professionals in acupuncture and moxibustion and have obtained Traditional Chinese Medicine practitioners. Before the beginning of the trial, they have been trained in the standard operating procedures for clinical studies organized by the research group. 
According to the relevant research, we chose three acupoints for moxibustion treatment, including ExLE4, ST35 and ST36[27, 28]. 30 minutes of treatment were delivered 3 times per week, for 4 weeks, with 12 sessions in total.

A total of two groups were designed in this trial, namely the experimental group (moxibustion and placebo gel group) and the control group (diclofenac sodium gel and placebo moxibustion group).

Experimental group used a moxa device invented by Professor Zhao of Beijing University of TCM[29]. The active moxibustion device had holes at the bottom to allow heat and smoke to radiate to the acupoints, but the placebo moxibustion device had a thermal insulation board over its bottom to isolate the smoke and most of the heat, preventing them from radiating to the skin.

In the course of the research, the participants was given a placebo gel (placebo gel In addition to the absence of an effective ingredient diclofenac sodium and no irritant or analgesic substances such as may affect the curative effect of the ingredients, and in other composition, appearance and odor is as same as therapeutic gel), and every time to take $4 \mathrm{~g}$ gel coating on each lesion knee, 3 times a day for 4 weeks. At the same time, the control group received $4 \mathrm{~g}$ diclofenac sodium gel applied to the diseased knee joint.

\section{Randomization and Blinding}

Randomization method adopted the central stochastic and data management of the subject group. The random number was generated by the SPSS19.0 statistical software, which was independent of the clinical trial statistics. The statisticians recorded random allocation cards with serial numbers, random numbers, and groups. Envelopes were encoded by serial number, sealed in opaque, and lined up in sequential order. After the participants met all the selection criteria and completed the baseline assessment, the researchers informed the center's statistician. The statistician opened the envelope based on the participant's screening number and then assigned the participant.

This was a double-blind trial, and neither the practitioner nor the participant knew about the assignment. Participants were treated in two separate rooms, and they were unaware of the task. Each treatment room has a designated doctor performing active or placebo moxibustion. The research coordinators at the participating hospitals did not participate in the treatment or evaluation, but only told the subjects which treatment room they needed to go for moxibustion. Similarly, evaluators and data analysts were unaware of the assignments throughout the research.

\section{Measurements}

Participants completed questionnaires at baseline, at the 2-and 4-week visits (the latter was the end of treatment), and at the 8-and 12-week follow-up visits (by telephone). The main outcome index is the change of total score of the Western Ontario and McMaster Universities Osteoarthritis Index (WOMAC) 
questionnaire, and the secondary outcome index includes WOMAC pain, stiffness, physiological function score, VAS score and PGA score[30]. Side effects of treatment, adverse events, and use of medication were also recorded. All data acquisition, processing, and analyses were performed by research staff who were blinded to group allocation.

\section{Sample Size}

According to the literature, a reduction of the WOMAC index at least $36 \%$ is effective[31] [32]. The significance level is $5 \%$ and statistical power is $90 \%$. With the dropout rate of $20 \%$, we planned to enroll 144 participants in the 2 groups, with 72 participants in each group.

\section{Statistical Analysis}

The description of baseline characteristics and clinical outcomes is based on the intended treatment population. Analysis was performed by SPSS software (SPSS 21.0, SPSS Inc, Chicago, IL, USA). The therapeutic effect on the outcome data was expressed as the difference in mean change, expressed as $95 \% \mathrm{Cl}$. P value $<0.05$ was considered statistically significant, and the test was bilateral. Population and baseline data were analyzed by standard descriptive statistics. We compared the WOMAC scale and VAS scores of the experimental group and the control group, and compared the two groups with the baseline. Non-parametric test ( $t$ test) was used to compare the difference of the average change of each result. Therapeutic effect changes between two groups were compared by the $\chi 2$ test. Analysts do not know that grouping is in all statistical analysis.

\section{Results}

From May 2016 to January 2018, 372 participants screened by telephone, and 183 attended the clinical screening. In all, 144 participants were recruited, and 139 were randomly assigned to treatment (figure 1). After 12 weeks, 5 participants ( 3 in the experimental group and 2 in the control group) had dropped out and were not contactable. Participants had a mean age of 59.0 years and $102(73.3 \%)$ were women. Table 1 presents the baseline characteristics of the participants with KOA. And 66 participants (48\%) had been diagnosed with KOA for 1 to $<5$ years. The groups were balanced at baseline (Table 1 ). 
Table 1

Baseline characteristics by study group

\begin{tabular}{|c|c|c|c|}
\hline & Experimental group & Control group & All Participants \\
\hline Characteristic & [n=69] & $\square n=70 \square$ & [n=1390 \\
\hline \multicolumn{4}{|c|}{ Demographic Characteristic } \\
\hline Female sex, No. (\%) & $51(73.9)$ & $51(72.9)$ & 102(73.3) \\
\hline Age, mean (SD), y & $57.6(10.5)$ & $60.3(9.9)$ & $59.0(10.2)$ \\
\hline Height, mean (SD), cm & $161.9(6.7)$ & $162.2(7.4)$ & 162.1(7.0) \\
\hline Weight, mean (SD), kg & $64.9(12.0)$ & 63.3(10.3) & $64.1(11.2)$ \\
\hline BMI, mean (SD) & $24.7(4.3)$ & $24.1(3.1)$ & $24.4(3.7)$ \\
\hline \multicolumn{4}{|c|}{ Symptom duration, No. (\%) } \\
\hline$<1 \mathrm{y}$ & $14(20)$ & 19(27) & $33(24)$ \\
\hline 1 to $<5 y$ & $39(57)$ & $27(39)$ & $66(48)$ \\
\hline 5 to $<10 y$ & 10(15) & $14(20)$ & $24(17)$ \\
\hline$\geqq 10 y$ & $6(9)$ & $10(14)$ & $16(12)$ \\
\hline \multicolumn{4}{|l|}{ Medication use, No. (\%) } \\
\hline Glucosamine products & $4(6)$ & $1(1)$ & $5(4)$ \\
\hline Analgesia & $1(1)$ & $0(0)$ & $1(1)$ \\
\hline \multicolumn{4}{|l|}{ Past treatment, No. (\%) } \\
\hline Moxibustion & $1(1)$ & $2(3)$ & $3(2)$ \\
\hline Acupuncture & 0 & $1(1)$ & $1(1)$ \\
\hline
\end{tabular}

Table 2 and 3 summarize continuous outcomes. Both the experimental group and the control group moderately relieved pain and stiffness and improved function compared with baseline, and the effect were maintained at 12 weeks. The analysis showed that there was no significant difference in all scores between the experimental group and the control group from 0 to 4 weeks (Table2 and 3, P>0.05). At 8 weeks, there were significant differences in WOMAC total score, pain score and functional score (Table2 and $3, P<0.05)$, indicating that the curative effect of the control group was better, while there were no significant differences in other scores (Table2 and 3, P > 0.05). At 12 weeks, there was no significant difference between the two groups (Table2 and $3, \mathrm{P}>0.05$ ). 
Table 2

Outcome Measurements During the Entire Study

$\begin{array}{lllll}\begin{array}{l}\text { Outcome } \\ \text { Measure }\end{array} & \begin{array}{l}\text { Experimental group } \\ (\mathrm{n}=69)\end{array} & \begin{array}{l}\text { Control group } \\ (\mathrm{n}=70)\end{array} & \begin{array}{l}\text { Mean } \\ \text { Difference }\end{array} & \text { P Value } \\ & & (95 \% \mathrm{Cl}) & \end{array}$

\section{WOMAC subscale score}

\section{Total score}

\begin{tabular}{|lllll}
\hline Baseline & $34.3(14.3)$ & $32.4(16.6)$ & $1.9(-3.3$ to 7.1$)$ & 0.47 \\
\hline 2 weeks & $26.1(13.0)$ & $26.3(16.3)$ & $-0.2(-5.2$ to 4.7$)$ & 0.93 \\
\hline 4 weeks & $22.5(13.7)$ & $19.4(13.6)$ & $3.1(-1.5$ to 7.7$)$ & 0.18 \\
\hline 8 weeks & $19.2(12.8)$ & $14.9 .8(9.9)$ & $4.3(0.5$ to 8.1$)$ & 0.03 \\
\hline 12 weeks & $17.4(12.5)$ & $14.4(9.0)$ & $3.0(-0.7$ to 6.6$)$ & 0.11 \\
\hline Pain & & & & \\
\hline Baseline & $7.4(3.3)$ & $7.0(3.3)$ & $0.4(-0.7$ to 1.5$)$ & 0.43 \\
\hline 2 weeks & $5.4(3.1)$ & $5.7(3.5)$ & $-0.3(-1.4$ to 0.8$)$ & 0.60 \\
\hline 4 weeks & $4.7(2.8)$ & $4.3(2.9)$ & $0.3(-0.6$ to 1.3$)$ & 0.23 \\
\hline 8 weeks & $4.1(2.6)$ & $3.1(2.4)$ & $1.0(0.2$ to 1.9$)$ & 0.02 \\
\hline 12 weeks & $3.7(2.7)$ & $3.1(2.2)$ & $0.6(-0.2$ to 1.5$)$ & 0.13 \\
\hline
\end{tabular}

\section{Stiffness}

\begin{tabular}{lllll} 
Baseline & $2.4(1.8)$ & $2.0(1.9)$ & $0.4(-0.2$ to 1.0$)$ & 0.24 \\
\hline 2 weeks & $1.7(1.5)$ & $1.4(1.4)$ & $0.3(-0.2$ to 0.8$)$ & 0.18 \\
\hline 4 weeks & $1.4(1.6)$ & $1.1(1.3)$ & $0.3(-0.2$ to 0.8$)$ & 0.30 \\
\hline 8 weeks & $1.1(1.3)$ & $0.8(1.1)$ & $0.3(-0.1$ to 0.7$)$ & 0.15 \\
\hline 12 weeks & $1.0(1.3)$ & $0.8(0.9)$ & $0.1(-0.2$ to 0.5$)$ & 0.45
\end{tabular}

\section{Function}

\begin{tabular}{lllll} 
Baseline & $24.5(10.4)$ & $23.4(12.8)$ & $1.1(-2.8$ to 5.0$)$ & 0.57 \\
\hline 2 weeks & $19.0(9.4)$ & $19.3(12.5)$ & $-0.3(-4.0$ to 3.4$)$ & 0.87 \\
\hline 4 weeks & $16.4(10.1)$ & $13.9(10.1)$ & $2.5(-0.9$ to 5.9$)$ & 0.15 \\
\hline 8 weeks & $13.9(9.6)$ & $11.0(7.1)$ & $3.0(0.1$ to 5.8$)$ & 0.04 \\
12 weeks & $12.7(9.4)$ & $10.5(6.7)$ & $2.2(-0.5$ to 4.9$)$ & 0.12
\end{tabular}




\begin{tabular}{|c|c|c|c|c|}
\hline Baseline & $3.6(1.2)$ & $3.6(1.2)$ & $0.0(-0.4$ to 0.4$)$ & 0.91 \\
\hline 2 weeks & $2.8(1.0)$ & $3.0(1.1)$ & $-0.1(-0.5$ to 0.2$)$ & 0.52 \\
\hline 4 weeks & $2.3(1.0)$ & $2.4(1.0)$ & $0.1(-0.4$ to 0.3$)$ & 0.61 \\
\hline 8 weeks & $2.2(1.0)$ & $2.3(1.0)$ & $0.0(-0.4$ to 0.3$)$ & 0.78 \\
\hline 12 weeks & $2.0(1.0)$ & $2.2(1.0)$ & $-0.2(-0.5$ to 0.2$)$ & 0.31 \\
\hline \multicolumn{5}{|c|}{$\begin{array}{l}\text { Abbreviations: WOMAC, Western Ontario and McMaster Universities Osteoarthritis Index; VAS, Visual } \\
\text { Analog Scale. The pain score is from } 0 \text { to } 20 \text {. The stiffness score is from } 0 \text { to } 8 \text {. The function score is } \\
\text { from } 0 \text { to } 68 \text {. The total score is from } 0 \text { to } 96 \text {. The VAS score is from } 0 \text { to } 10 \text {. The higher score } \\
\text { indicates worse function. }\end{array}$} \\
\hline \multicolumn{5}{|c|}{ Experimental group and control group data are expressed as mean (SD) unless stated otherwise. } \\
\hline
\end{tabular}


Table 3

PGA at follow-up, Values are No. (\%) of participants unless stated otherwise

\begin{tabular}{|c|c|c|c|}
\hline Outcome Measure & Experimental group ( $n=72)$ & Control group $(n=72)$ & $\mathrm{P}$ Value \\
\hline \multicolumn{4}{|l|}{2 week } \\
\hline Much improved & $14(19)$ & $14(19)$ & \multirow[t]{5}{*}{0.36} \\
\hline Minimally improved & $49(69)$ & $43(61)$ & \\
\hline No change & $8(11)$ & $14(19)$ & \\
\hline Minimally worse & 0 & 0 & \\
\hline Much worse & 0 & 0 & \\
\hline Missing data & $1(1)$ & $1(1)$ & I \\
\hline \multicolumn{4}{|l|}{4 week } \\
\hline Much improved & $15(21)$ & $21(30)$ & \multirow[t]{5}{*}{0.50} \\
\hline Minimally improved & $49(69)$ & $43(61)$ & \\
\hline No change & $5(6)$ & $5(6)$ & \\
\hline Minimally worse & 0 & $1(1)$ & \\
\hline Much worse & 0 & 0 & \\
\hline Missing data & $3(4)$ & $2(2)$ & l \\
\hline \multicolumn{4}{|l|}{8 week } \\
\hline Much improved & $19(27)$ & $19(27)$ & \multirow[t]{5}{*}{0.84} \\
\hline Minimally improved & $41(57)$ & $44(61)$ & \\
\hline No change & $9(12)$ & $7(10)$ & \\
\hline Minimally worse & 0 & 0 & \\
\hline Much worse & 0 & 0 & \\
\hline Missing data & $3(4)$ & $2(2)$ & / \\
\hline \multicolumn{4}{|l|}{12 week } \\
\hline Much improved & $21(30)$ & $18(26)$ & \multirow[t]{5}{*}{0.45} \\
\hline Minimally improved & $39(54)$ & $43(60)$ & \\
\hline No change & $9(12)$ & $7(10)$ & \\
\hline Minimally worse & 0 & $2(2)$ & \\
\hline Much worse & 0 & 0 & \\
\hline
\end{tabular}




\section{Adverse events}

Two participants (1 in the experimental group and 1 in the control group) reported adverse events within 12 weeks (Table 4). One participant in the experimental group developed blisters caused by moxibustion in the fourth week and recovered from adverse events. One participant in the control group developed localized skin papules, mainly associated with diclofenac sodium gel, and recovered after stopping treatment. Both participants recovered from adverse events and did not withdraw from the trial. Therefore, the two interventions in this trial were safe for treating KOA subjects.

Table 4

Adverse events

\begin{tabular}{|lll|}
\hline & Experimental group & Control group \\
\cline { 2 - 3 } Adverse events & $\square \mathrm{n}=69 \square$ & $\square \mathrm{n}=70[$ \\
\hline Skin pimples & 0 & 1 \\
\hline Skin blisters & 1 & 0 \\
\hline Skin paresthesia & 0 & 0 \\
\hline Systemic allergy & 0 & 0 \\
\hline
\end{tabular}

\section{Discussion}

This research is a double-blinded, double-placebo, randomized controlled trial evaluate the difference of efficacy between moxibustion and local diclofenac sodium gel in the treatment of KOA. This trial showed that the therapeutic effect of moxibustion and topical diclofenac sodium gel persisted from the end of treatment to the next 8 weeks. From 0 to 4 weeks, moxibustion has the same therapeutic effect as topical diclofenac sodium gel, which can alleviate varying degrees of pain, stiffness and dysfunction of KOA participants. At week 8 , local diclofenac sodium gel was more effective than moxibustion in reducing pain and dysfunction. That is to say, the long-term effect of local diclofenac sodium gel may be better than moxibustion. The explanation of it is that some participants kept their gel and carried on using it after the treatment.

Moxibustion is often used in the treatment of arthritis in the clinic. A large number of randomized controlled trials results show that moxibustion can effectively alleviate pain in participants with KOA, improve dysfunction, and improve the quality of life of participants[21] [22]. 
Blindness is used to exclude biases caused by expectations and subjective assessments. The absence of blind design in most clinical studies is a common problem, leading to high risk of bias in test results [23]. Therefore, the efficacy of moxibustion in KOA are still controversial. However, it is difficult to achieve complete blindness in moxibustion clinical trials, especially comparing moxibustion and drugs[33]. We believe that direct comparison of moxibustion and drugs may have some limitations during the trial. Therefore, few outcome evaluators and participants can avoid the effects of subjective bias in the assessment. Therefore, in order to reduce the bias of clinical randomized controlled trials and improve the quality of evidence-based medical raw data, this research used a double-blinded, double-placebo design to compare the efficacy of moxibustion and topical diclofenac sodium gel in the treatment of knee osteoarthritis.

The "double placebo" design method was used in clinical trials for the first time to compare the efficacy of indomethacin and phenylbutazone in the treatment of rheumatic diseases in clinical trials [34]. Wang applied the double placebo design method in acupuncture clinical trials for the first time to compare the clinical efficacy of acupuncture and flunarizine in the treatment of migraine [35].

Our trial was a double-blinded, double-placebo trial that reduced the effects of psychological factors and maintained the same conditions throughout the trial. We applied the new moxibustion device invented to implementation of active / placebo in a blinded moxibustion [25]. The placebo moxibustion device resembled the active one in appearance, except the bottom of the device. The experimental group used active moxibustion and the control group used placebo moxibustion. Participants in the control group received a diclofenac sodium gel, while a placebo gel was administered to the experimental group. The placebo gel was identical in composition to the diclofenac sodium gel except that diclofenac sodium was not present. Both treatments have the same appearance and texture.

The placebo moxibustion method made the control subjects unable to feel the thermal stimulation, light radiation stimulation and other therapeutic stimuli of the moxibustion at the moxibustion site, thereby achieving the effect of placebo. This was the most critical issue in the design of placebo moxibustion devices. In this study, neither the practitioner nor the participant knew about the grouping. Since the operation of moxibustion and placebo moxibustion was similar to that of the participant, there was no formal examination of the success rate of the blind method or the expected value of the participant. However, a similar shedding rate (3 to 2 participants) indicated that the participant had successfully blinded and the treatment in both groups is equally convincing.

In our experiments, the efficacy of moxibustion was the same as most trials, suggesting that the efficacy of moxibustion was not worse than standard drugs, and that the efficacy was only slightly worse than standard drugs in the long-term. However, local diclofenac sodium gel has some adverse reactions, such as local skin reactions, rash, drug-sensitive dermatitis, application-site erythema, skin irritation, application-site paresthesia and dry skin [36-40]. Therefore, moxibustion has an advantage in controlling adverse reactions. 
This study used the osteoarthritis evaluation scale WOMAC, and also used VAS, PGA and other evaluation indicators, but lacked an assessment of the quality of life of participants. At the same time, the above indicators are subjective measurement results, and the objective indicators such as serum biomarkers or X-rays are not designed. In the adverse events, we did not record some events such as dry skin that Chinese people did not pay much attention to in the past. An assessment of such evaluation indicators can be added in the future.

In summary, trials had shown that the therapeutic effect of moxibustion on KOA was not worse than local diclofenac sodium gel, which differed only in long-term efficacy. The results of this trial provided evidence for the efficacy of moxibustion on pain relief and improvement in physical function in participants with KOA. The results also can provide reliable raw data for systematic reviews and evidence-based medicine.

\section{Declarations}

\section{Ethics approval and consent to participate}

The following ethical review committee has reviewed and approved this study. The protocol (version 2.0, date 17 November 2015) was approved the IRB/EC of the Teaching Hospital of Chengdu University of TCM (referece:2015KL-014). The study was also registered with the National Institutes of Health (NIH) Clinical Trials Registry in May 2016 (website: Clinical Trials.gov). Registration number: NCT02772055. This trial will be conducted according to the principles of the Declaration of Helsinki (version Edinburgh 2000). All participants will be informed about the purpose of the trial and its risks and benefits, and written informed consent will be obtained by the local study coordinator prior to entry into the trial.

\section{Consent for publication}

Consent for publication from the participants to report individual patient data has been obtained from each participant.

\section{Availability of data and materials}

Not applicable.

\section{Competing interests}

The authors declare that they have no competing interests.

\section{Funding}




\section{Authors' contributions}

Ling Luo, Mo Liao and Jian-ying Zhou contributed equally to this work. Ling Luo, Mo Liao and Jian-ying Zhou wrote the manuscript and performed the statistical analysis. Jian-ying Zhou, Lin-lin Zhu, and Ling Luo wrote the study protocol. Jia-xi Peng, Cai-rong Zhang, Ling-ling Guo, Min Liu, Jia-ping Wang, Xiao-xia Han, Sheng-feng Lu, Tian-shu Hou, Ping Du, Jing-Jing Zhao, Jia Zuo, Jing-zhu Chen, Bi-qing Huang, Jia Peng, Guo-ping Ran performed the trial. Shu-guang Yu, Yong Tang, Hai-yan Yin, Qiao-feng Wu, ChengShun Zhang and Peng Lv reviewed the manuscript and provided critical feedback and approval for submission. Yong Tang and Shu-guang Yu conceived and designed the trial and had final responsibility for the decision to submit for publication. All authors read and approved the final manuscript.

\section{Acknowledgements}

The authors would like to acknowledge the cooperation of the participants in this trial.

\section{Authors' information (optional)}

Not applicable.

\section{References}

1.Litwic, A., et al., Epidemiology and burden of osteoarthritis. Br Med Bull, 2013. 105: p. 185-99.

2. Guidelines for the diagnosis and treatment of Osteoarthritis (2007 edition) \%J Chinese Medical Journal. 2008(01): p. 28-30.

3.Antman, E. M., et al., Use of nonsteroidal antiinflammatory drugs: an update for clinicians: a scientific statement from the American Heart Association. Circulation, 2007. 115(12): p. 1634-42.

4.Silverstein, F. E., et al., Gastrointestinal toxicity with celecoxib vs nonsteroidal anti-inflammatory drugs for osteoarthritis and rheumatoid arthritis: the CLASS study: A randomized controlled trial. Celecoxib Long-term Arthritis Safety Study. JAMA, 2000. 284(10): p. 1247-55.

5.Whelton, A., Nephrotoxicity of nonsteroidal anti-inflammatory drugs: physiologic foundations and clinical implications. Am J Med, 1999. 106(5B): p. 13S-24S.

6.Wolfe, M. M., D. R. Lichtenstein, and G. Singh, Gastrointestinal toxicity of nonsteroidal antiinflammatory drugs. N Engl J Med, 1999. 340(24): p. 1888-99. 
7.Zhang, W., et al., OARSI recommendations for the management of hip and knee osteoarthritis: part III: Changes in evidence following systematic cumulative update of research published through January 2009. Osteoarthritis Cartilage, 2010. 18(4): p. 476-99.

8.Bookman, A. A., K. S. Williams, and J. Z. Shainhouse, Effect of a topical diclofenac solution for relieving symptoms of primary osteoarthritis of the knee: a randomized controlled trial. CMAJ, 2004. 171(4): p. $333-8$.

9.Conaghan, P. G., et al., A multicentre, randomized, placebo- and active-controlled trial comparing the efficacy and safety of topical ketoprofen in Transfersome gel (IDEA-033) with ketoprofen-free vehicle (TDT 064) and oral celecoxib for knee pain associated with osteoarthritis. Rheumatology (Oxford), 2013. 52(7): p. 1303-12.

10.Komatsu, T. and T. Sakurada, Comparison of the efficacy and skin permeability of topical NSAID preparations used in Europe. Eur J Pharm Sci, 2012. 47(5): p. 890-5.

11.van Haselen, R. A. and P. A. Fisher, A randomized controlled trial comparing topical piroxicam gel with a homeopathic gel in osteoarthritis of the knee. Rheumatology (Oxford), 2000. 39(7): p. 714-9.

12.Cheng, C. W., et al., Extending the CONSORT Statement to moxibustion. J Integr Med, 2013. 11(1): p. $54-63$.

13.Huang, Q., et al., Bibliometric Analysis of Diseases Spectrum of Moxibustion Therapy \% J Journal of Acupuncture and Tuina Science. 2012. 10(06): p. 342-348.

14.Zhao, L., et al., Effectiveness of moxibustion treatment as adjunctive therapy in osteoarthritis of the knee: a randomized, double-blinded, placebo-controlled clinical trial. Arthritis Res Ther, 2014. 16(3): p. R133.

15.Ren, X., et al., Effectiveness of moxibustion treatment in quality of life in patients with knee osteoarthritis: a randomized, double-blinded, placebo-controlled trial. Evid Based Complement Alternat Med, 2015. 2015: p. 569523.

16.Li, C., Y. Han, and B. Dong, Effect of acupuncture moxibustion on RANTES and MCP-1 expression of knee osteoarthritis \% J Journal of Liaoning University of Traditional Chinese Medicine. 2013. 15(10): p. $79-81$.

17.Xiong, Y., R. Peng, and S. Xia, Expression of interleukin $1 \beta$ and tumor necrosis factor alpha in articular fluid of knee osteoarthritis in rabbits and its effect on moxibustion \% J Chinese Organization Engineering Research. 2010. 14(41): p. 7700-7703.

18.Tan, C., et al., Preliminary correlation between warm needling treatment for knee osteoarthritis of deficiency-cold syndrome and metabolic functional genes and pathways. J Acupunct Meridian Stud, 2010. 3(3): p. 173-80. 
19.Zhou, Z., et al., Therapeutic effect of separating moxibustion on blood stasis type knee osteoarthritis \%J Shanghai Journal of Acupuncture and Moxibustion. 2010. 29(01): p. 45-47.

20.Sun, K., J. Yang, and D. K. Shen, [Clinical observation on treatment of primary knee osteoarthritis of liver and kidney deficiency type with Aconite cake-separated moxibustion]. Zhongguo Zhen Jiu, 2008. 28(2): p. 87-90.

21.Kim, T. H., et al., Moxibustion treatment for knee osteoarthritis: a multi-centre, non-blinded, randomised controlled trial on the effectiveness and safety of the moxibustion treatment versus usual care in knee osteoarthritis patients. PLoS One, 2014. 9(7): p. e101973.

22.Song, G. M., et al., Moxibustion is an Alternative in Treating Knee Osteoarthritis: The Evidence From Systematic Review and Meta-Analysis. Medicine (Baltimore), 2016. 95(6): p. e2790.

23.Choi, T. Y., et al., Moxibustion for the treatment of osteoarthritis: An updated systematic review and meta-analysis. Maturitas, 2017. 100: p. 33-48.

24.MacPherson, H., et al., Revised STandards for Reporting Interventions in Clinical Trials of Acupuncture (STRICTA): Extending the CONSORT statement. J Evid Based Med, 2010. 3(3): p. 140-55.

25.Zhou, J. Y., et al., Moxibustion versus diclofenac sodium gel for the treatment of knee osteoarthritis: a study protocol for a double-blinded, double-placebo, randomised controlled trial. BMJ Open, 2017. 7(4): p. e012879.

26. Recommendations for the medical management of osteoarthritis of the hip and knee: 2000 update. American College of Rheumatology Subcommittee on Osteoarthritis Guidelines. Arthritis Rheum, 2000. 43(9): p. 1905-15.

27.Chen, N., et al., Study on the law of Acupuncture and moxibustion treatment of KOA using the data mining \% J Chinese Journal of TCM Information. 2016. 23(03): p. 31-34.

28.Yang, H., S. Chen, and J. Li, Study on acupuncture and moxibustion treatment of knee joint osteoarthritis with data mining \%J Chinese Journal of TCM Information. 2017. 24(01): p. 95-98.

29.Zhao, B., et al., A novel sham moxibustion device: a randomized, placebo-controlled trial. Complement Ther Med, 2006. 14(1): p. 53-60; discussion 61.

30.Bellamy, N., et al., Validation study of WOMAC: a health status instrument for measuring clinically important patient relevant outcomes to antirheumatic drug therapy in patients with osteoarthritis of the hip or knee. J Rheumatol, 1988. 15(12): p. 1833-40.

31.Altman, R., et al., Development of criteria for the classification and reporting of osteoarthritis. Classification of osteoarthritis of the knee. Diagnostic and Therapeutic Criteria Committee of the American Rheumatism Association. Arthritis Rheum, 1986. 29(8): p. 1039-49. 
32.in Osteoarthritis: Care and Management in Adults. 2014: London.

33.Dawson, J., et al., Impact of persistent hip or knee pain on overall health status in elderly people: a longitudinal population study. Arthritis Rheum, 2005. 53(3): p. 368-74.

34.Marusic, A. and S. F. Ferencic, Adoption of the double dummy trial design to reduce observer bias in testing treatments. J R Soc Med, 2013. 106(5): p. 196-8.

35.Wang, L. P., et al., Efficacy of acupuncture for migraine prophylaxis: a single-blinded, double-dummy, randomized controlled trial. Pain, 2011. 152(8): p. 1864-71.

36.Baraf, H. S., et al., Safety and efficacy of topical diclofenac sodium 1\% gel in knee osteoarthritis: a randomized controlled trial. Phys Sportsmed, 2010. 38(2): p. 19-28.

37.Stockfleth, E., et al., Phase IV head-to-head randomized controlled trial comparing ingenol mebutate $0.015 \% \mathrm{gel}$ with diclofenac sodium $3 \% \mathrm{gel}$ for the treatment of actinic keratosis on the face or scalp. $\mathrm{Br} \mathrm{J}$ Dermatol, 2018. 178(2): p. 433-442.

38.Moreira, S. A. and D. J. Liu, Diclofenac systemic bioavailability of a topical 1\% diclofenac $+3 \%$ menthol combination gel vs. an oral diclofenac tablet in healthy volunteers: a randomized, open-label, crossover study. Int J Clin Pharmacol Ther, 2017. 55(4): p. 368-372.

39.Wadsworth, L. T., J. D. Kent, and R. J. Holt, Efficacy and safety of diclofenac sodium 2\% topical solution for osteoarthritis of the knee: a randomized, double-blind, vehicle-controlled, 4 week study. Curr Med Res Opin, 2016. 32(2): p. 241-50.

40.Nelson, C., et al., Phase IV, open-label assessment of the treatment of actinic keratosis with 3.0\% diclofenac sodium topical gel (Solaraze). J Drugs Dermatol, 2004. 3(4): p. 401-7.

\section{Figures}




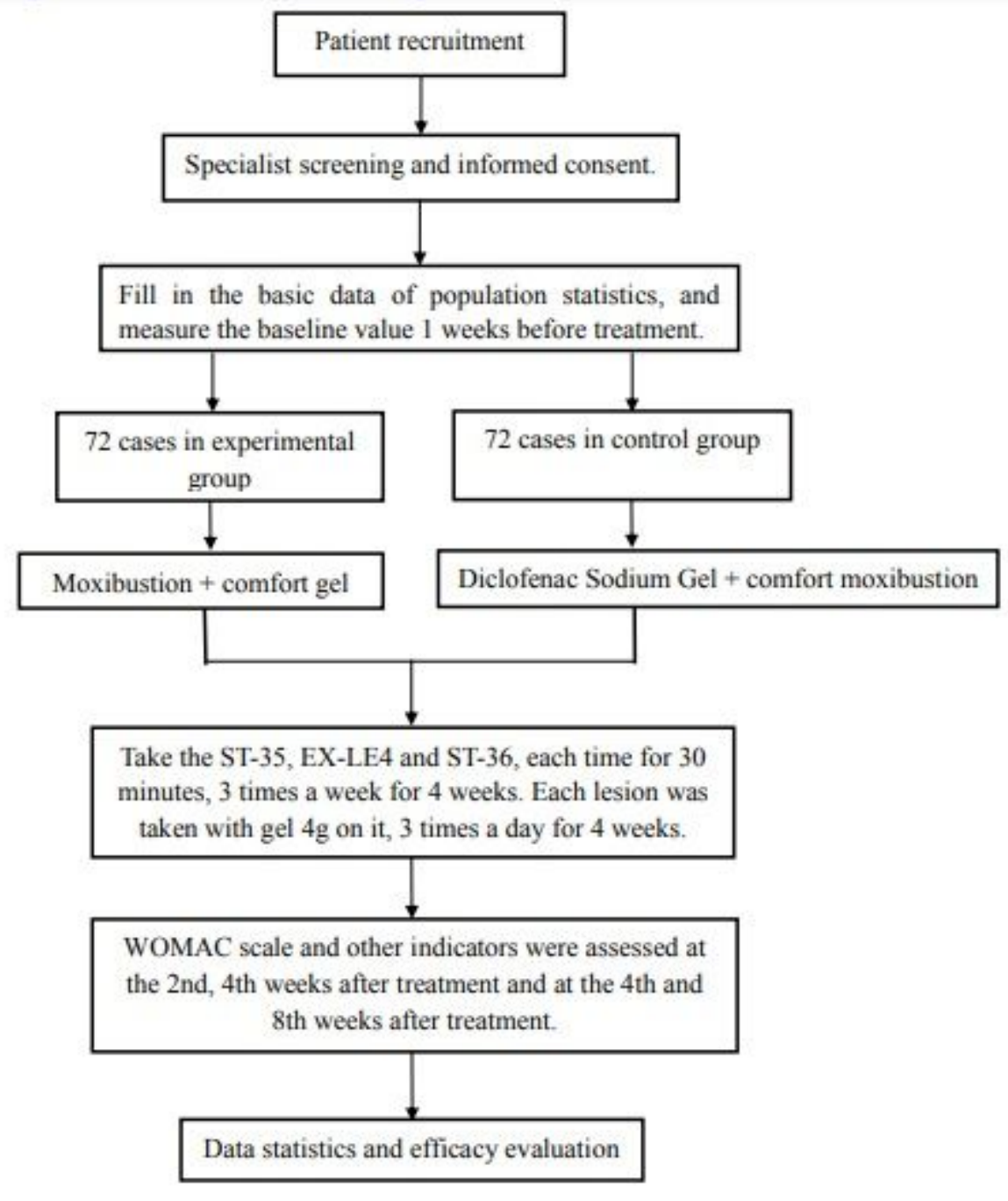

Figure 1

Flow of Participants Through the Trial

\section{Supplementary Files}

This is a list of supplementary files associated with this preprint. Click to download.

- CONSORT2010Checklist.doc 